\title{
Proteomics analysis and proteogenomic characterization of different physiopathological human lenses
}

\author{
Xiaohang $\mathrm{Wu}^{1 \dagger}$, Zhenzhen $\mathrm{Liu}^{1 \dagger}$, Xiayin Zhang ${ }^{1 \dagger}$, Dongni Wang ${ }^{1 \dagger}$, Erping Long ${ }^{1}$, Jinghui Wang ${ }^{1}$, Wangting $\mathrm{Li}^{1}$,
} Weiyi Lai', Qianzhong Cao ${ }^{1}$, Kunhua Hư ${ }^{2}$, Weirong Chen ${ }^{1}$, Haotian Lin ${ }^{1 *}$ and Yizhi Liu ${ }^{1 *}$

\begin{abstract}
Background: The aim of the present study was to identify the proteomic differences among human lenses in different physiopathological states and to screen for susceptibility genes/proteins via proteogenomic characterization.

Methods: The total proteomes identified across the regenerative lens with secondary cataract (RLSC), congenital cataract (CC) and age-related cataract (ARC) groups were compared to those of normal lenses using isobaric tagging for relative and absolute protein quantification (iTRAQ). The up-regulated proteins between the groups were subjected to biological analysis. Whole exome sequencing (WES) was performed to detect genetic variations.

Results: The most complete human lens proteome to date, which consisted of 1251 proteins, including 55.2\% previously unreported proteins, was identified across the experimental groups. Bioinformatics functional annotation revealed the common involvement of cellular metabolic processes, immune responses and protein folding disturbances among the groups. RLSC-over-expressed proteins were characteristically enriched in the intracellular immunological signal transduction pathways. The CC groups featured biological processes relating to gene expression and vascular endothelial growth factor (VEGF) signaling transduction, whereas the molecular functions corresponding to external stress were specific to the ARC groups. Combined with WES, the proteogenomic characterization narrowed the list to 16 candidate causal molecules.

Conclusions: These findings revealed common final pathways with diverse upstream regulation of cataractogenesis in different physiopathological states. This proteogenomic characterization shows translational potential for detecting susceptibility genes/proteins in precision medicine.
\end{abstract}

Keywords: Human lens, Regenerative lenses with secondary cataract (RLSC), Proteomics, Proteogenomic analysis

\section{Background}

The eye lens has the most abundant protein content within the body, with proteins accounting for more than $35 \%$ of its wet weight [1]. The disruption of the normal quality or quantity of lens constitutional proteins leads to the opacification of the refractive structure, namely, cataracts, which are the leading cause of blindness worldwide [2]. Cataracts can be induced under a variety of physiopathological states, including (but not limited

\footnotetext{
*Correspondence: haot.lin@hotmail.com; yizhi_liu@aliyun.com

${ }^{\dagger}$ Equal contributors

'State Key Laboratory of Ophthalmology, Zhongshan Ophthalmic Center, Sun Yat-sen University, 54\# Xianlie Road, Guangzhou, Guangdong 510060, China

Full list of author information is available at the end of the article
}

to) age-related cataracts (ARCs) and congenital cataracts (CCs). Although different types of cataracts share common final alterations of lens crystallin proteins in terms of their quality (structure) and quantity [3, 4], the complicated predisposing factors (including genetics and environmental stimuli) and the upstream regulatory mechanisms are highly diverse and have not been thoroughly studied. Genetic alterations account for less than 30\% percent of cataractogenesis events in CCs, and far fewer genetic associations have been detected in ARCs [5].

In a recent study, we achieved functional lens regeneration in $\mathrm{CC}$ patients after a novel minimally invasive surgical procedure [6]. The included subjects were tested in advance to exclude cataractogenetic genomic variants. 
Unfortunately, three cases still exhibited evident secondary opacification of the regenerative lenses more than 2 years after primary surgery. The unique regenerative lens with secondary cataract (RLSC) model has enable the identification of a novel proteome composition and the characterization of a novel molecular mechanism underlying the predisposition to cataractogenesis $[7,8]$.

An overwhelming majority of previous cataract proteomics studies have exclusively focused on crystallin proteins; thus, little is known about non-crystallin proteins, which are expressed at significantly lower levels but still play key roles in the maintenance of lens function [9]. Isobaric tagging for relative and absolute protein quantification (iTRAQ) is a recently developed technology for quantitative proteomics that enables for high-throughput identification and relative quantification of trace amounts of protein [10]. Moreover, the widely performed whole exome sequencing (WES) methodology can identify numerous genetic variants as potential origins of protein alterations [11, 12]. By combining these techniques, this proteogenomic characterization is expected to narrow the list of candidate causal molecules. Therefore, we conducted a preliminary proteomics comparative analysis of RLSC, CC and ARC with respect to different physiopathological states, using iTRAQ, followed by WES of representative individuals. These findings will update our current knowledge of the molecular mechanism responsible for cataractogenesis and lens development. Furthermore, predictive therapeuticdirected pathways or molecules may be discerned by combining the genetic and proteomics perspectives.

\section{Methods}

\section{Sample collection}

The regenerative, $\mathrm{CC}$ and $\mathrm{ARC}$ lenses were obtained from the Zhongshan Ophthalmic Center (ZOC) in Guangzhou, China. As a group of control, normal human lenses were obtained from the Eye Bank of Guangdong Province. The study protocol conformed to the ethical guidelines of the
1975 Declaration of Helsinki and was approved by the Ethical Review Committee of ZOC. Written informed consent was obtained from all subjects (or from at least one guardian of each participating child).

$\mathrm{CC}$ and ARC subjects were examined and confirmed by at least three experienced ophthalmologists according to the International Classification of Diseases and Codes. The lens contents were collected during cataract extraction.

The regenerative lenses were obtained using novel minimally invasive surgical methods of cataract removal developed by our group, which preserved the endogenous lens epithelial stem cells (LECs) and produced regenerative lenses in situ with improved visual function. Unfortunately, three cases exhibited evident secondary total opacification of the regenerative lenses beyond 2 years after primary surgery. Thus, secondary cataract extraction surgery was performed as a routine approach (irrigation and lens aspiration [I/A] with no phacoemulsification energy administered) with intraocular lens implantation, during which the regenerated lens contents were collected. The baseline information of the included subjects is presented in Table 1 . All samples were immediately stored at $-80^{\circ} \mathrm{C}$ until further analysis.

\section{Sample preparation and iTRAQ labelling (Fig. 1)}

Eight lens samples from four groups were processed and individually examined. Protease inhibitor (Roche Complete ULTRA tablets, mini EASY pack) was added to each sample prior to the experiments. Cold acetone was subsequently added at 4 times the volume of the pellet, and the sample was precipitated at $-20{ }^{\circ} \mathrm{C}$ overnight. The sample was subsequently centrifuged twice at $14,000 \mathrm{x} \mathrm{g}$ for $10 \mathrm{~min}$ at $4{ }^{\circ} \mathrm{C}$. Subsequently, the pellet was air-dried, and protein lysis was performed in lysis buffer. The proteins were quantified using the GE 2-D Quant Kit according to the manufacturer's instructions. Each sample containing $100 \mu \mathrm{g}$ of quantified protein was enzymolyzed with trypsin and labelled with iTRAQ reagent using the iTRAQ Reagent-8Plex Multiplex Kit

Table 1 Baseline information of the included subjects

\begin{tabular}{|c|c|c|c|c|c|c|}
\hline Group & Sample No. & $\begin{array}{l}\text { Age } \\
\text { (years) }\end{array}$ & Gender & Cataract morphology & iTRAQ labelling & WES \\
\hline \multirow[t]{3}{*}{ RLSC } & 1 & 2 & Male & NA & 113 & Yes \\
\hline & 2 & 2 & Male & NA & 114 & Yes \\
\hline & 3 & 2 & Female & NA & 115 & No \\
\hline \multirow[t]{3}{*}{ CC } & 4 & 2 & Male & Nuclear & 116 & Yes \\
\hline & 5 & 2 & Male & Nuclear & 117 & No \\
\hline & 6 & 3 & Female & Nuclear & 118 & No \\
\hline ARC & 7 & 63 & Female & $\begin{array}{l}\text { Nuclear + cortical } \\
\text { (LOCS II) }\end{array}$ & 119 & No \\
\hline Normal lens & 8 & 21 & Male & NA & 121 & No \\
\hline
\end{tabular}

RLSC regenerative lenses with secondary cataract, $C C$ congenital cataract, $A R C$ aged-related cataract, WES whole exome sequencing 


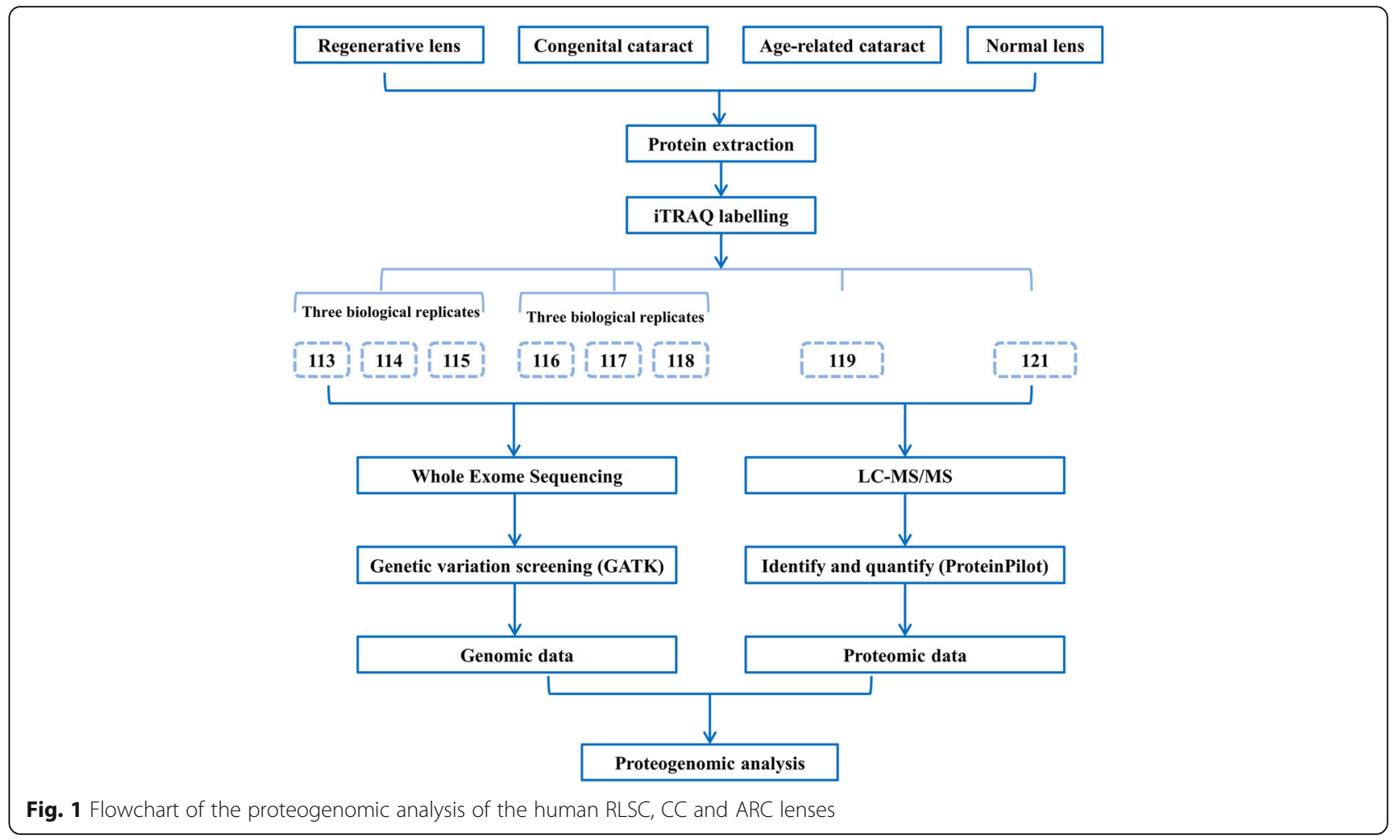

(AB SCIEX 4381663) according to the manufacturer's instructions. The three regenerative lenses were labelled with iTRAQ reagents 113,114 , and 115; the three CC lenses were labelled with iTRAQ reagents 116,117 , and 118; and the ARC and normal lenses were labelled with iTRAQ reagents 119 and 121, respectively. Figure 1 illustrates the workflow of the detailed experiment procedures.

\section{Liquid chromatography tandem mass spectrometry (LC-MS/MS) and data analysis}

The protein samples were separately measured using the Dionex Ultimate 3000 RSLCnano system. The pre-column was washed with the $0.1 \%$ formic acid and $2 \%$ acetonitrile. In each measurement, the peptides from a standard sample were loaded offline onto a $75-\mu \mathrm{m}$ i.d. $\times 150 \mathrm{~mm}$ Acclaim PepMap RSLC C18 column $(2 \mu \mathrm{m}, 100 \AA$ A, nanoViper). The flow was diverted to the separation column at a flow rate of $300 \mathrm{~nL} / \mathrm{min}$. The peptides were separated and eluted with an effective gradient of $4-50 \%$ solution B ( $80 \%$ acetonitrile $[\mathrm{ACN}]$ and $0.1 \%$ formic acid) in a 65 -min survey full scan, and the five most intense ions were selected for a zoom scan to determine the charge state using the Thermo Scientific Q Exactive Hybrid QuadrupoleOrbitrap $^{\text {tw }}$ Mass Spectrometer. Subsequently, MS/MS with higher-energy collisional dissociation (HCD) scans were acquired in an Orbitrap at a resolution of 17,500; the maximum ion accumulation time and target value were maintained at $60 \mathrm{~ms}$ and $4 \mathrm{e}^{5}$, respectively, and the normalized collision energy (NCE)/ stepped NCE was 30.

ProteinPilot $^{\text {tm }} 5.0$ software (AB SCIEX, Framingham, MA, USA, 2012), Paragon $^{\text {Tm }}$ Algorithm 5.0.0.0, 4767 was subsequently used for protein identification and iTRAQ quantification. The database searching parameters were set as follows: trypsin as the digestion enzyme; iTRAQ 8plex as the sample type; no special factors; biological modification as ID focus; thorough as search effort; HUMAN_uniprot_2015.8.3.fasta as the database; false discovery rate analysis was conducted, and the detected protein threshold was set at less than 0.01; the detected protein threshold [Unused ProtScore (Conf)] cutoff was 1.3 (unused ProtScore), with at least two peptides with 95\% confidence.

The identified proteins were statistically analyzed to filter for the deregulated proteins of each group compared to normal lens. The filtering steps include: 1) the difference between groups were compared using t-test and $p<0.05$ was considered statistically significant; 2) the coefficient of variation (C.V) was used to evaluate the dispersion of the replicates within groups, and detected proteins with $\mathrm{C} . \mathrm{V} \leq 0.5$ were considered reliable; 3) proteins with ratio of $>20$ or $<0.5$ were considered generated with unreliable signals and excluded; 4) protein ratios of $\geq 2$ and $\leq 0.5$ represented significant up-regulation and down-regulation between the groups. 
Biological interpretation of the protein expression data The significantly up-regulated proteins in each group were annotated according to the Gene Ontology (GO) database (https://david-d.ncifcrf.gov/summary.jsp) [13]. Pathway annotation of these proteins was conducted by searching against the Kyoto Encyclopedia of Genes and Genomes (KEGG) database (http://www.genome.jp/ kegg/pathway.html). The significance of the association between the dataset and canonical pathways was measured as the ratio of the protein number from the dataset that maps to the canonical pathway and the $p$-value of the estimate. A functional regulatory network analysis of the common evaluated proteins from the three groups was performed using the web-based Search Tool for the Retrieval of Interacting Genes/Proteins (STRING) database (version 10.0, http://string.embl.de/). STRING was used to build the diagrams from numerous sources, including known experimental data and/or computational data based on genomic context analysis. The matching interactions were provided with a confidence score based on the reliability of the interaction.

\section{WES and data analysis}

The genomic DNA from the blood samples of two subjects in the RLSC group and one subject in the CC group was extracted using Qiagen blood midi or maxi kits (Qiagen, Hilden, Germany) according to the manufacturer's instructions. WES was performed at iGenostics Biotechnology (Guangzhou, China). All samples were processed using the Illumina HiSeq 2000 platform.

The human reference genome was GRCh37/hg19, and the RefGene database was downloaded from the University of California, Santa Cruz (UCSC). Read mapping and polymerase chain reaction (PCR) duplicate removal were performed using the Burrows-Wheeler Aligner (BWA), Sequence Alignment Map (SAM) Tools and Picard tools. The Genome Analysis Toolkit (GATK) was used for local realignment, base quality score recalibration and variant calling (HaplotypeCaller) with default parameters. Single-nucleotide polymorphism (SNP) and insertion or deletion (INDEL) variants were annotated using ANNOVAR. The candidate variations were manually reviewed using the Integrative Genomics Viewer (IGV).

\section{Results}

The most complete proteome identification of human lenses and comprehensive quantification of the distinguished expressed proteins in different physiopathological states

In total, 1251 proteins were identified across all lens samples, based on the detected protein threshold set at $p<0.05$. A complete list of proteins identified with detailed information regarding their identification and quantification is provided in Additional file 1: Table S1.
Compared to their abundance in the normal lens (control), the abundances of the 261,202, and 177 proteins identified in the RLSC, CC and ARC groups, respectively, were significantly upregulated. (protein ratio $\geq 2$, $\mathrm{C} . \mathrm{V} \leq 0.5$ ), and 80 proteins were found in the overlapping areas. Most of the significantly up-regulated proteins in the CC (95.5\%) and ARC (86.4\%) groups overlapped, whereas the up-regulated proteins in the RLSC group was were more specific, with a lower overlapping percentage (73.6\%). A preponderance of downregulated proteins (156) was found, and the percentage of specific proteins $(32.1 \%)$ in the RLSC groups was higher than those in the ARC $(78,59.0 \%)$ and CC (53, $32.1 \%$ ) groups, as presented in the Venn diagram in Fig. 2a.

An overview of previous reports on the identified proteins and the up-regulated components in each group of human lenses is presented in Fig. 2b. A large majority of the proteins $(55.2 \%, 691 / 1251)$ identified in the total protein pool have not previously been reported. Among the minor reported components, proteins identified using MudPIT constituted the largest proportion (92.3\%, 517/560), followed by two-dimensional (2-D) LC-MS/ MS identification [3] $(30.9 \%, 173 / 560)$, and by iTRAQ [14] identification $(21.1 \%, 118 / 560)$, National Center for Biotechnology Information (NCBI) GenBank records $(9.3 \%, 51 / 560)$ and UniProtKB records $(4.3 \%, 24 / 560)$. The RLSC $(22.6 \%, 156 / 691)$, CC $(18.7 \%, 129 / 691)$, and ARC $(16.8 \%, 116 / 691)$ groups exhibited a degressive, unreported protein ratio.

\section{Bioinformatics functional annotation revealed common final pathways with diverse upstream regulation for cataractogenesis in different physiopathological states} The GO annotation analyses were performed for the 261, 202, and 177 significantly up-regulated proteins of the RLSC, CC and ARC groups, respectively, to elucidate their biological processes, molecular functions and cellular components.

\section{GO biological process analysis}

Cellular protein metabolic process, immune responsive process and protein folding-related biological processes were the top enriched items shared by the RLSC $(n=32$, 22 , and 16 , respectively; all $p<0.0001), \mathrm{CC}(n=37,19$, and 15 , respectively; all $p<0.0001$ ) and ARC groups ( $n=37,16$, and 14 , respectively; all $p<0.0001$ ). In the RLSC group, the Fc-epsilon receptor signaling pathway was primarily involved $(n=10, p=0.027)$, followed by Ras protein signal transduction $(n=10, p=0.005)$ and Antigen processing and presentation of peptide antigen via major histocompatibility complex (MHC) class I $(n=8, p=0.004)$. In the CC group, gene expression $(n=23, p=0.005)$, the epidermal growth factor receptor 

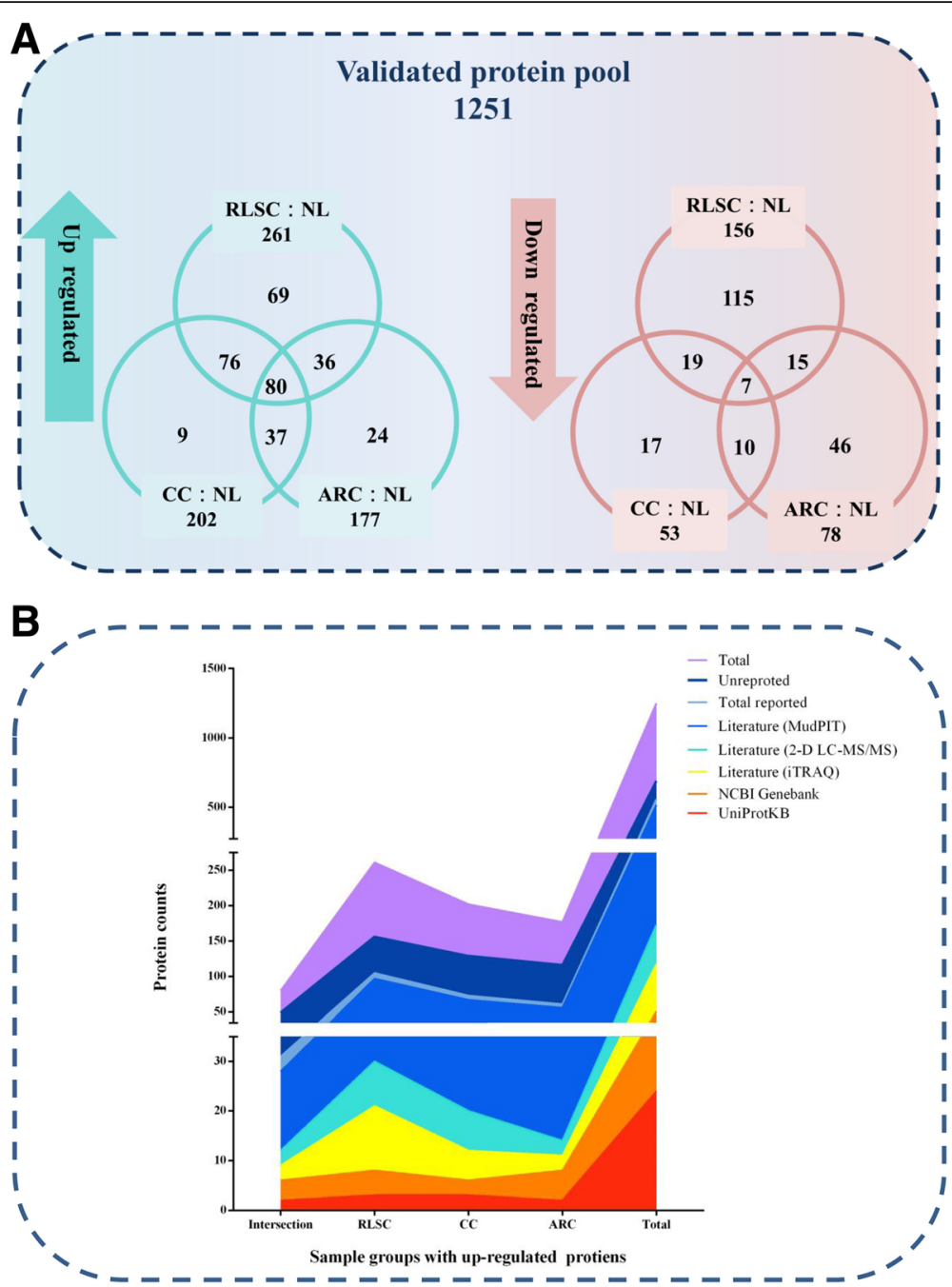

Fig. 2 Differentially expressed proteins identified across the human RLSC, CC, ARC groups and an overview of previous reports. a. Venn diagram of the inter-relationship of the proteins identified in the human RLSC, CC and ARC groups. b. Overview of previous reports on proteins identified in human lenses

signaling pathway $(n=11, p=0.008)$, and the cellular $n i-$ trogen compound metabolic process $(\mathrm{n}=10, p=0.026)$ were the most predominantly enriched items. In contrast, in the ARC group, enrichment of the items response to drug $(\mathrm{n}=11, p=0.007)$, response to hypoxia $(\mathrm{n}=8$, $p=0.006)$ and response to endoplasmic reticulum stress ( $n=7, p=0.0002)$ was observed (Fig. 3a).

\section{GO molecular function analysis}

The most significant enriched GO molecular function items shared by the RLSC $(n=169,47$, and 23 , respectively; all $p<0.0001)$, CC ( $n=161,47$, and 21, respectively; all $\mathrm{p}<0.0001)$ and ARC $(n=158,37$, and 17 , respectively; all $p<0.0001)$ groups were protein binding, poly (A) RNA binding and protein homodimerization activity, respectively. In the RLSC group, calcium ion binding was predominantly involved $(n=24, p<0.0001)$, followed by beta-catenin binding $(n=7, p=0.0007)$ and MRNA
3'-UTR binding $(n=4, p=0.020)$. In CC group, enzyme binding $(n=13, p=0.0009)$, the structural constituents of ribosome $(n=8, p=0.017)$, and calmodulin binding $(\mathrm{n}=7$, $p=0.025)$ were the most predominantly enriched items. Finally, in the ARC group, syntaxin binding $(n=5$, $p=0.015)$, nicotinamide adenine dinucleotide (NAD) binding $(\mathrm{n}=4, p=0.011)$ and oxygen transporter activity ( $n=3, p=0.012)$ were observed (Fig. 3b).

\section{GO cellular component analysis}

The overwhelming majority of the enriched proteins were localized in the extracellular exosome, cytoplasm, membrane and the extended substructures in the RLSC ( $n=132$, 92, and 60 , respectively; all $p<0.0001), \mathrm{CC}$ ( $n=121,95$, and 66, respectively; all $p<0.0001$ ) and ARC $(n=119,79$, and 68 , respectively; all $p<0.0001)$ groups. In particular, the cellular component item 


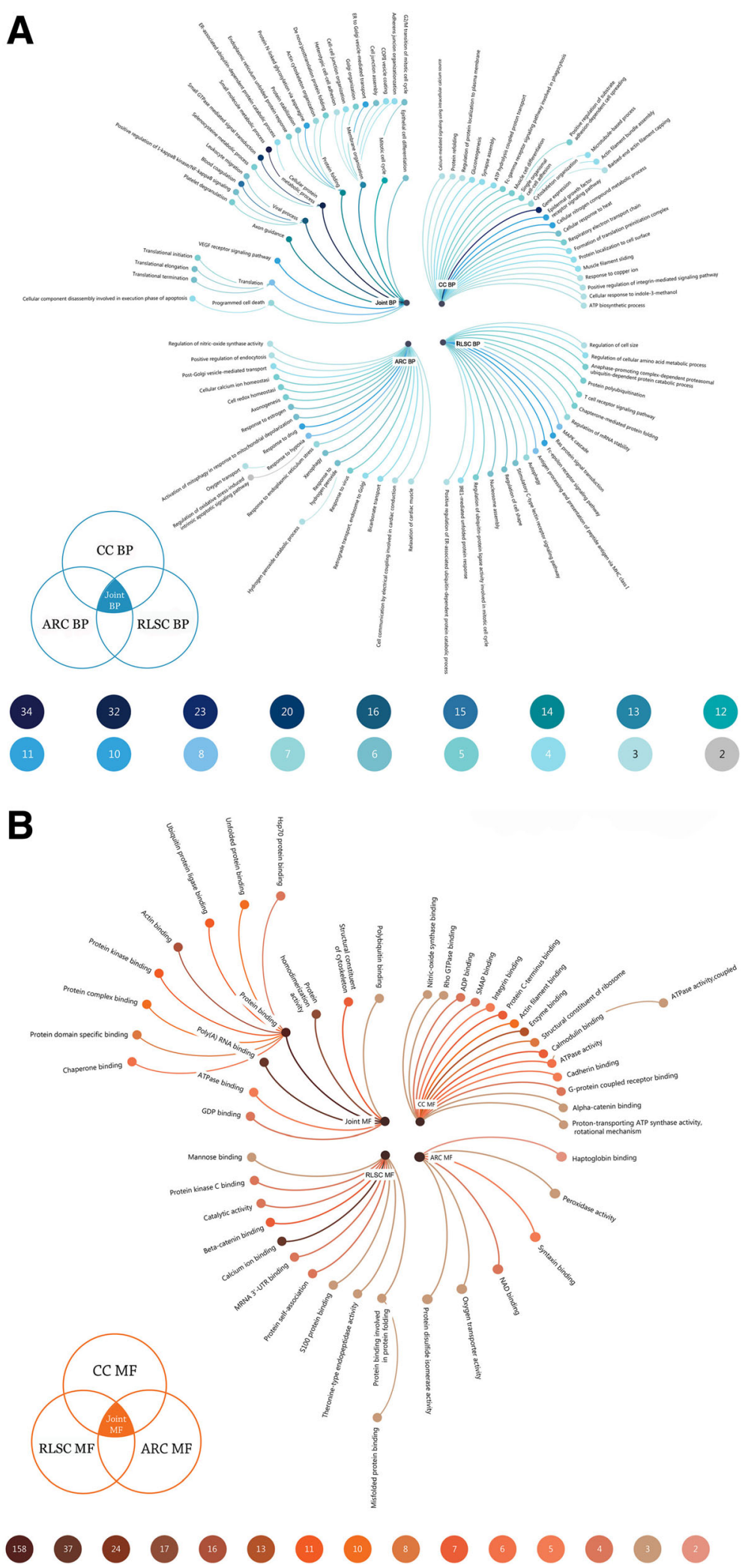

Fig. 3 Radial tidy trees illustrating the significant GO items involved in the human RLSC, CC, and ARC groups and their joint components. The node texts represent the involved GO terms; the node colour gradients reflect the degree of enrichment of the corresponding GO terms. $\mathbf{a}$. The significantly involved GO biological process (BP) items; b. The significantly involved GO molecular function (MF) items 
nucleus was involved in the RLSC $(n=79, p=0.038)$ and CC $(n=74, p=0.0374)$ groups but was not significantly involved in the ARC group.

\section{KEGG analysis}

KEGG pathway mapping revealed 10, 22 and 17 enriched pathways in the RLSC, CC and ARC groups, respectively. These groups shared the three most commonly affected pathways: protein processing in the endoplasmic reticulum $(n=16,12$, and 13; all $p<0.0001)$, phagosome $(\mathrm{n}=11,15$, and 12; all $p<0.0001)$ and regulation of the actin cytoskeleton (all $\mathrm{n}=16 ; p=0.006,0.009$, and 0.006). In the RLSC group, Huntington's disease $(n=8, p=0.031)$ and proteasome ( $\mathrm{n}=8, p=0.031)$ were specifically involved. In the CC group, Focal adhesion $(n=9, p=0.022)$, Leukocyte transendothelial migration $(n=8, p=0.004)$, and ribosome ( $n=8, p=0.025)$ were the most dominant enriched items. Finally, in the ARC groups, Endocytosis ( $n=9, p=0.048$ ), Salivary secretion $(n=6, p=0.010)$ and Proximal tubule bicarbonate reclamation ( $n=4, p=0.005$ ) were specifically observed (Table 2).

\section{The unbalanced abundance of the representative} cataractous proteins with relatively high sequence coverage between groups provides insights into the underlying molecular mechanisms in different physiopathological states Comparing the relative abundances of the principal proteins between groups and the normal lens as a control, $\alpha$-crystallin proteins showed no changes in different physiopathological conditions; $\beta-, \gamma-$ and $\lambda$ crystallin protein families were broadly down-regulated, except for $\beta$-crystallin A2, which exhibited significant overexpression in the RLSC, CC and ARC groups.

Cytoskeletal proteins, including beaded filament structural protein (BFSP1), BFSP2, tubulin alpha 1a (TUBA1A), myosin heavy chain 9 (MYH9) and retinitis pigmentosa 2 (RP2), were predominantly up-regulated proteins in the ARC group but were less prominent in the RLSC and CC groups. Vimentin was prominently over-expressed in CC lenses. Thus, dysfunction, rather than inadequate amounts of these components, contributes to the intracellular cytoskeletal instability in the corresponding physiopathological states.

Abhydrolase domain containing 12 (ABHD12), which is a crucial metabolic enzyme, was most significantly overexpressed in the RLSC group, followed by the CC and ARC groups. Other metabolic enzymes, including lens protein with glutamine synthetase domain (LGSN), aldehyde dehydrogenase 1 family member A1 (ALDH1A1), apolipoprotein A1 (APOA1), sorbitol dehydrogenase (SORD), tyrosine 3-monooxygenase/tryptophan 5-monooxygenase activation protein epsilon (YWHAE+), apolipoprotein E (APOE), and lanosterol synthase (LSS), were down-regulated or slightly up-regulated in the RLSC, CC and ARC groups.
Membrane proteins, including peroxisomal biogenesis factor 14 (PEX14), integrin subunit beta 1 (ITGB1), junctional adhesion molecule 3 (JAM3), integral membrane protein 2B (ITM2B), gap junction protein alpha (GJA3), GJA8, and wolframin ER transmembrane glycoprotein (WFS1), were generally up-regulated in the RLSC, CC and ARC groups. Further observation revealed that PEX14, ITGB1 and JAM3 were prominently up-regulated in the RLSC groups, indicating the potential involvement of intercellular adhesion in the regulation of lens regeneration. See Fig. 4 and Table 3 for details.

The protein-protein interaction network generated by the identified cataract-associated proteins in different physiopathological states

Using the STRING interaction database, the commonly up-regulated proteins shared by the RLSC, CC, and ARC groups were analyzed to illustrate the protein-protein interactions associated with cataractogenesis. Mediumconfidence interactions (minimum required interaction score, 0.400 ) were set for the analysis. The common upregulated proteins primarily comprised the protein networks, indicating the involvement of protein folding, response to stress and phagosome (Fig. 5).

\section{Proteogenomic characterization narrowed the list of candidate causal molecules}

WES testing and analysis were performed for two individuals in the RLSC group and one individual in the CC group (Additional file 2: Table S2). After bioinformatics filtering from the raw sequencing data based on mutation types, low minor allele frequencies and functional predictions, 160 variants of the corresponding 125 genes were prioritized for further proteogenomic interpretation. Among the 125 variant genes, 16 corresponding proteins were simultaneously detected and validated using iTRAQ. In the RLSC group, 11 proteins were detected with the corresponding genetic variants, among which 3 proteins (peroxiredoxin [PRX], heterogeneous nuclear ribonucleoprotein D like [HNRNPDL] and WFS1) were significantly up-regulated (fold-change $>2$ ); another 3 proteins (fatty acid synthase [FASN], transportin 1 [TNPO1] and keratin 10[KRT10]) were significantly down-regulated (fold-change $<0.5$ ). In the CC group, 6 proteins were detected with the corresponding genetic variants, among which 2 proteins (carbonic anhydrase 2 [CA2] and dolichyl-phosphate mannosyltransferase subunit 1 [DPM1]) were significantly upregulated (fold-change $>2$ ); another 2 proteins (ubiquitin c-terminal hydrolase L1 [UCHL1] and crystallin gamma D [CRYGD]) were significantly down-regulated (foldchange $<0.5$ ) (Table 4 and Fig. 6). 
Table 2 The KEGG pathways involved in RLSC, CC and ARC by the up-regulated proteins

\begin{tabular}{|c|c|c|c|}
\hline & KEGG pathways & Gene counts & $p$-value \\
\hline \multirow[t]{10}{*}{ RLSC group } & Protein processing in endoplasmic reticulum & 16 & $<0.0001$ \\
\hline & Phagosome & 11 & 0.0001 \\
\hline & Regulation of actin cytoskeleton & 10 & 0.0060 \\
\hline & Tight junction & 6 & 0.0330 \\
\hline & Bacterial invasion of epithelial cells & 5 & 0.0346 \\
\hline & Salmonella infection & 5 & 0.0420 \\
\hline & Pathogenic Escherichia coli infection & 5 & 0.0084 \\
\hline & Huntington's disease & 8 & 0.0313 \\
\hline & Proteasome & 4 & 0.0319 \\
\hline & Cell adhesion molecules (CAMs) & 8 & 0.0071 \\
\hline \multirow[t]{23}{*}{ CC group } & Phagosome & 15 & $<0.0001$ \\
\hline & Protein processing in endoplasmic reticulum & 12 & 0.0001 \\
\hline & Tight junction & 11 & $<0.0001$ \\
\hline & Pathogenic Escherichia coli infection & 11 & $<0.0001$ \\
\hline & Regulation of actin cytoskeleton & 10 & 0.0090 \\
\hline & Bacterial invasion of epithelial cells & 8 & 0.0003 \\
\hline & Salmonella infection & 6 & 0.0127 \\
\hline & Focal adhesion & 9 & 0.0225 \\
\hline & Leukocyte transendothelial migration & 8 & 0.0036 \\
\hline & Ribosome & 8 & 0.0072 \\
\hline & Parkinson's disease & 8 & 0.0098 \\
\hline & Hippo signaling pathway & 8 & 0.0130 \\
\hline & Hypertrophic cardiomyopathy (HCM) & 6 & 0.0099 \\
\hline & Fc gamma R-mediated phagocytosis & 6 & 0.0133 \\
\hline & Gap junction & 6 & 0.0160 \\
\hline & Legionellosis & 5 & 0.0126 \\
\hline & Proteoglycans in cancer & 11 & 0.0018 \\
\hline & Arrhythmogenic right ventricular & & $<0.0001$ \\
\hline & cardiomyopathy (ARVC) & 10 & \\
\hline & Cell adhesion molecules (CAMs) & 8 & 0.0098 \\
\hline & Dilated cardiomyopathy & 7 & 0.0028 \\
\hline & Vibrio cholerae infection & 5 & 0.0118 \\
\hline & Shigellosis & 5 & 0.0223 \\
\hline \multirow[t]{18}{*}{ ARC group } & Protein processing in endoplasmic reticulum & 13 & $<0.0001$ \\
\hline & Phagosome & 12 & $<0.0001$ \\
\hline & Regulation of actin cytoskeleton & 10 & 0.0057 \\
\hline & Pathogenic Escherichia coli infection & 8 & $<0.0001$ \\
\hline & Bacterial invasion of epithelial cells & 7 & 0.0013 \\
\hline & Tight junction & 7 & 0.0084 \\
\hline & Salmonella infection & 6 & 0.0095 \\
\hline & Endocytosis & 9 & 0.0480 \\
\hline & Salivary secretion & 6 & 0.0100 \\
\hline & Proximal tubule bicarbonate reclamation & 4 & 0.0053 \\
\hline & Vasopressin-regulated water reabsorption & 4 & 0.0294 \\
\hline & $\begin{array}{l}\text { Endocrine and other factor-regulated calcium } \\
\text { reabsorption }\end{array}$ & 4 & 0.0330 \\
\hline & Proteoglycans in cancer & 9 & 0.0122 \\
\hline & Shigellosis & 5 & 0.0177 \\
\hline & Dilated cardiomyopathy & 5 & 0.0424 \\
\hline & Vibrio cholerae infection & 5 & 0.0093 \\
\hline & Arrhythmogenic right ventricular & & 0.0249 \\
\hline & cardiomyopathy (ARVC) & 5 & \\
\hline
\end{tabular}




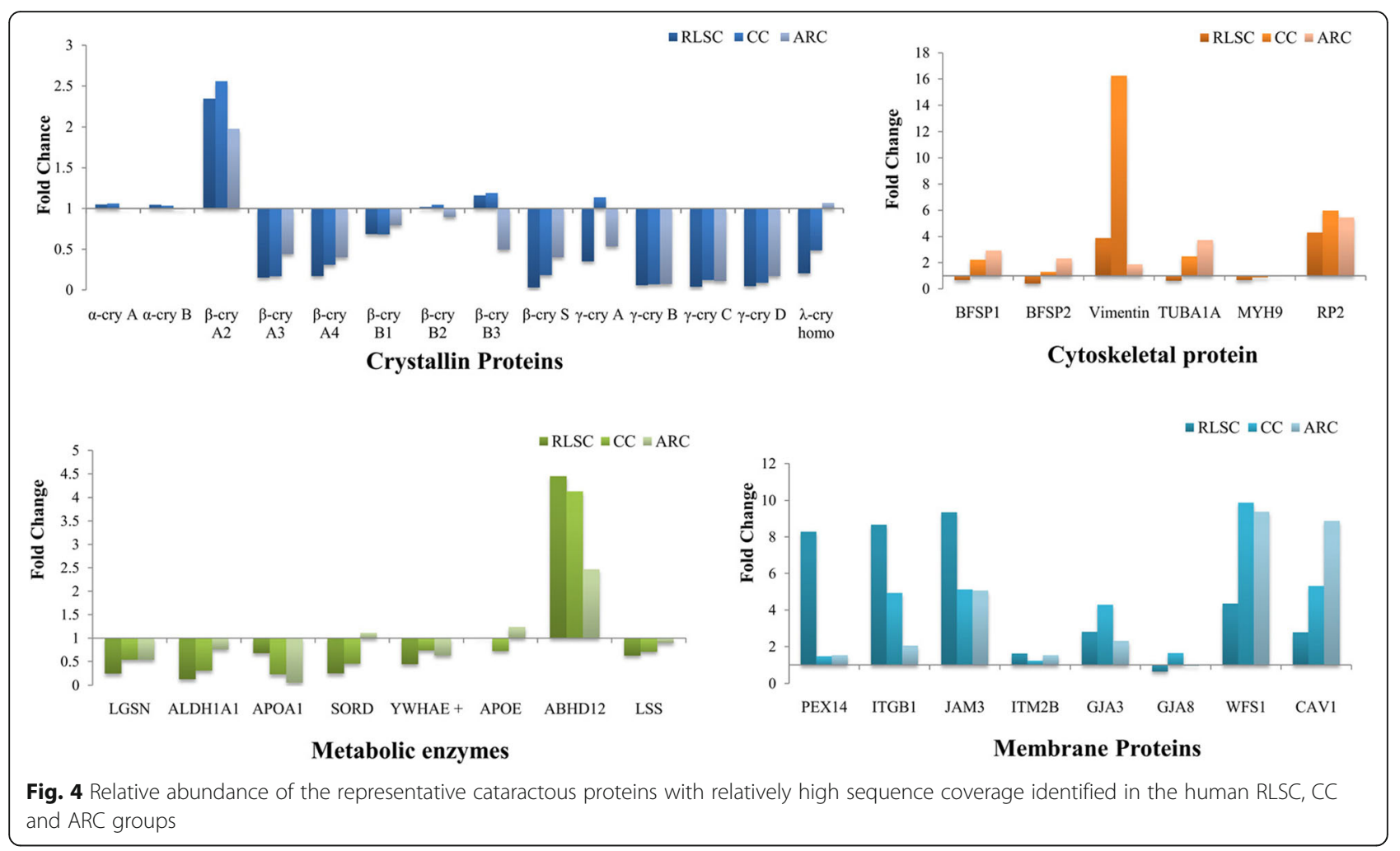

\section{Discussion}

The present study focused on the quantitative comparison of the human lens proteomic data obtained from different physiopathological states (i.e., the RLSC, CC and ARC groups). The most complete human lens proteome, including 1251 proteins, was identified, and a large majority of the proteins were previously unreported. Bioinformatics functional annotation revealed the common involvement of cellular metabolic process, immune responses and protein folding disturbances, but diverse upstream regulation were observed for cataractogenesis in different physiopathological states. Combined with the detection of genetic variants using WES, the susceptibility genes/proteins were narrowed to a list of 16 molecules.

The differentially expressed proteins identified in the RLSC, CC and ARC groups overlapped in relatively large proportions, indicating the involvement of common underlying biological processes. Subsequently, the performance and interpretation of the GO analysis, KEGG pathways and STRING networks defined the common final pathways by extracting the common items among the individual groups. The common final biological processes for RLSC, congenital and senile cataracts were inferred to enable cellular metabolic processes, immune responses and protein folding disturbances. The underlying molecular mechanisms were multilevel, including transcriptional modification (poly [A] RNA binding) and post-translational protein interventions (protein binding and protein homodimerization activity). The main location of the common cataractogenesis function was predicted to be extranuclear zones, such as the extracellular exosome, cytoplasm, and membrane.

Interestingly, the RLSC, CC and ARC processes follow diverse paths but arrive at the same destination. The mysterious regulation of lens regeneration was associated with immune responses, and evidence of an enriched Fc-epsilon receptor signaling pathway, antigen processing and presentation of peptide antigens via MHC class I was found. Unlike the common final pathways, the specific process disposal component was indicated in the nucleus, with cellular signal transduction regulation by calcium ion and ubiquitinproteasome pathways.

The biological processes of the CC group were associated with gene expression and vascular endothelial growth factor (VEGF) signaling pathways, confirming the involvement of genetic predisposition and development dysregulation [15]. Age-related cataractogenesis featured responses to external stimuli, including hypoxia, drugs, estrogen, and viruses. In contrast to RLSC and CC, ARC involved specific processes localized to the extracellular area with rare interruption of the genetic materials. As previously suggested, this finding could indicate that fetal lenses, as in the RLSC and CC groups, contain more cells with relatively complete cellular structures, including the nucleus and organelles, and 
Table 3 Relative abundances of the representative cataractous proteins identified in the RLSC, CC and ARC groups

\begin{tabular}{|c|c|c|c|c|c|}
\hline Category & Protein & UniProt ID & RLSC AVG & CC AVG & ARC AVG \\
\hline \multirow[t]{14}{*}{ Crystallin genes } & Alpha-crystallin A chain & P02489 & 1.050 & 1.060 & 1.000 \\
\hline & Alpha-crystallin B chain & E9PR44 & 1.045 & 1.035 & 0.991 \\
\hline & Beta-crystallin A2 & P53672 & 2.345 & 2.558 & 1.977 \\
\hline & Beta-crystallin A3 & P05813 & 0.152 & 0.169 & 0.441 \\
\hline & Beta-crystallin A4 & P53673 & 0.171 & 0.309 & 0.402 \\
\hline & Beta-crystallin B1 & P53674 & 0.688 & 0.682 & 0.794 \\
\hline & Beta-crystallin B2 & P43320 & 1.017 & 1.044 & 0.895 \\
\hline & Beta-crystallin B3 & P26998 & 1.161 & 1.190 & 0.497 \\
\hline & Beta-crystallin S & P22914 & 0.029 & 0.183 & 0.402 \\
\hline & Gamma-crystallin A & P11844 & 0.352 & 1.137 & 0.535 \\
\hline & Gamma-crystallin B & P07316 & 0.059 & 0.071 & 0.072 \\
\hline & Gamma-crystallin C & P07315 & 0.038 & 0.122 & 0.112 \\
\hline & Gamma-crystallin D & P07320 & 0.047 & 0.088 & 0.171 \\
\hline & Lambda-crystallin homologue & Q9Y2S2 & 0.206 & 0.487 & 1.067 \\
\hline \multirow[t]{8}{*}{ Membrane protein } & Peroxisomal membrane protein PEX14 & O75381 & 8.287 & 1.473 & 1.528 \\
\hline & Integrin beta- 1 & P05556 & 8.663 & 4.931 & 2.051 \\
\hline & Junctional adhesion molecule C & Q9BX67 & 9.345 & 5.120 & 5.058 \\
\hline & Integral membrane protein $2 \mathrm{~B}$ & Q9Y287 & 1.614 & 1.229 & 1.528 \\
\hline & Gap junction alpha-3 protein & Q9Y6H8 & 2.800 & 4.291 & 2.312 \\
\hline & Gap junction alpha-8 protein & P48165 & 0.640 & 1.641 & 0.946 \\
\hline & Wolframin & O76024 & 4.350 & 9.872 & 9.376 \\
\hline & Caveolin-1 & Q03135 & 2.785 & 5.311 & 8.872 \\
\hline \multirow[t]{6}{*}{ Cytoskeletal protein } & BFSP1 (Filensin) & Q12934 & 0.675 & 2.212 & 2.911 \\
\hline & BFSP2 (Phakinin) & Q13515 & 0.408 & 1.291 & 2.333 \\
\hline & Vimentin & $\mathrm{P} 08670$ & 3.864 & 16.248 & 1.871 \\
\hline & Tubulin alpha-1A chain & Q71U36 & 0.632 & 2.466 & 3.733 \\
\hline & Myosin-9 & P35579 & 0.681 & 0.865 & 0.964 \\
\hline & Protein XRP2 & O75695 & 4.291 & 5.970 & 5.445 \\
\hline \multirow[t]{8}{*}{ Metabolic enzymes } & Lengsin & Q5TDP6 & 0.242 & 0.538 & 0.535 \\
\hline & Retinal dehydrogenase 1 & P00352 & 0.130 & 0.304 & 0.759 \\
\hline & Apolipoprotein A-I & P02647 & 0.678 & 0.225 & 0.057 \\
\hline & Sorbitol dehydrogenase & Q00796 & 0.247 & 0.452 & 1.107 \\
\hline & 14-3-3 protein epsilon & P62258 & 0.443 & 0.737 & 0.631 \\
\hline & Apolipoprotein E & P02649 & 1.001 & 0.725 & 1.236 \\
\hline & Monoacylglycerol lipase ABHD12 & Q8N2K0 & 4.448 & 4.122 & 2.466 \\
\hline & Lanosterol synthase & P48449 & 0.625 & 0.709 & 0.895 \\
\hline
\end{tabular}

Green represents the up-regulated proteins and red indicates the down-regulated proteins

therefore occupy the biological process and molecular pathways involving the DNA and RNA within the nucleus [3].

The expression of most crystallin proteins, including $\alpha$, $\beta$, and $\gamma$ crystallin proteins was down-regulated in regenerative lens, congenital and senile cataracts compared with that in normal lenses, consistent with the findings of $\mathrm{Su}$ et al. [16]. The involved mechanism was suggested to be the evident aggregation [17, 18] and degradation [19] of crystallins during cataract formation. An exception was $\beta$-crystallin $\mathrm{A}_{2}$, which exhibited significant up-regulation in the RLSC, CC and ARC groups with the normal lens as the control. $\beta$-crystallin $\mathrm{A}_{2}$ was not included in the identification of human crystallins in previous proteomics reports. The dynamic expression of the crystallin components and its implications remains elusive.

$\mathrm{CC}$ is a representative disease with substantial genetic heterogeneity and manifested complexity [20, 21]. Genomic sequencing failed to detect the causal genetic variants in sporadic patients, who constitute more than two thirds of the total number. After linking the genome to the proteome in the present study, hundreds of candidate genetic variants and thousands of differentially expressed proteins were eliminated to create a list of 16 genes and their corresponding proteins. Thus, this methodology is useful for simplifying the complexity of isolated genomic or proteomics data and could support the development of precision medicine, particularly for diseases with high genetic and clinical heterogeneity [22].

\section{Study strengths and limitations}

The present study should be interpreted within the context of its strengths and limitations. The unique RLSC samples, the most complete human lens proteome identification to date and the comprehensive multilayer bioinformatics interpretations demonstrate not only the strength but also some of the limitations inherent to the present study. The regenerative lenses were obtained from our 


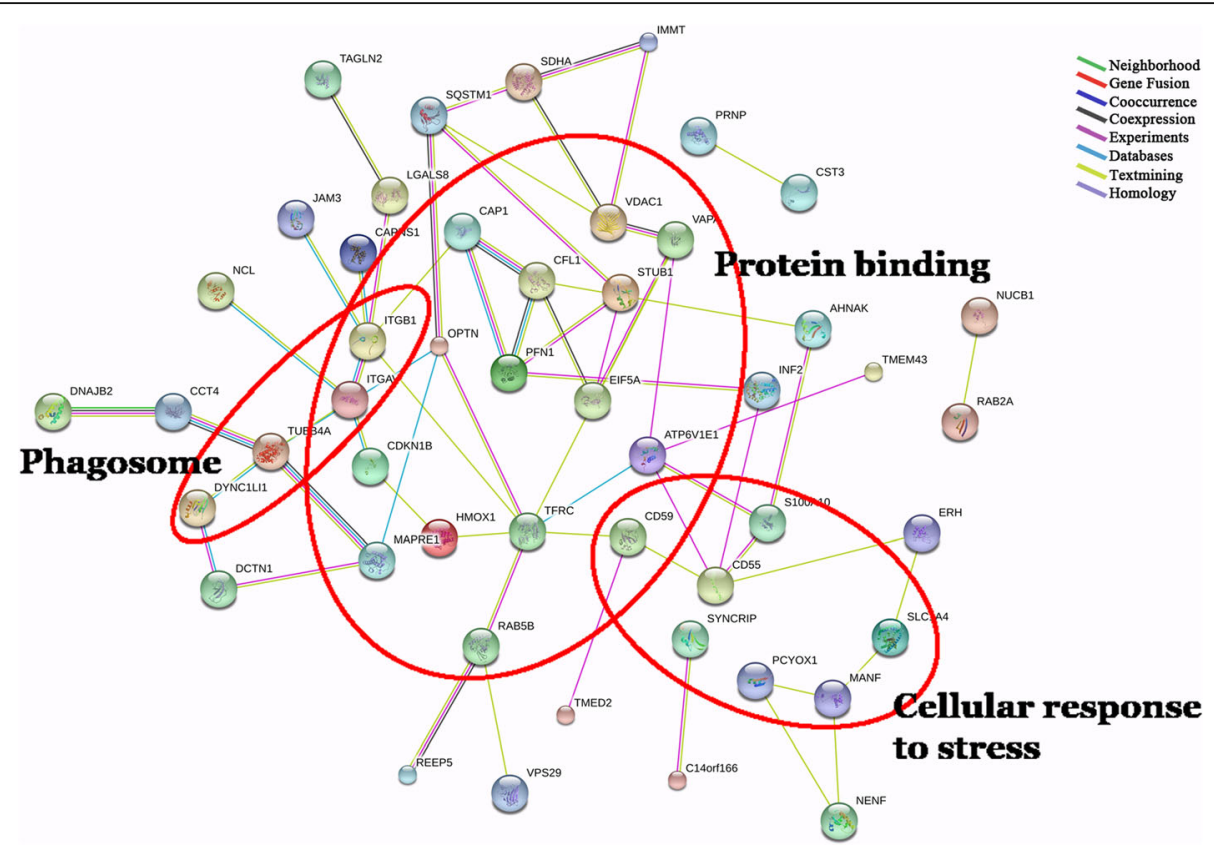

Fig. 5 Protein-protein interaction network of the identified cataract-associated proteins in different physiopathological states

novel minimally invasive surgical procedures, and transparency was maintained in most subjects. Thus, the number of samples in the RLSC group was too small, and experimental replicates or validations were not feasible. Additionally, all samples were collected during minimally invasive surgical procedures. However, it was not realistic to obtain the entire lens contents or the specific lens zone subjected to extracapsular cataract extraction (ECCE).

Nevertheless, because a quality control strategy was used for proteomics identification and because each surgery was performed by an experienced surgeon, the findings contribute to elucidating the mechanism underlying cataractogenesis with respect to diverse crystallin lens dysregulated models. Subsequent studies involving larger sample sizes and experimental validation will be conducted to confirm and further explore the target molecular pathways. Moreover, the proteogenomic analysis conducted here has great favorable translational potential for detecting candidate susceptibility genes/proteins and, thus, facilitating the development of precision medicine, particularly for diseases with high genetic and clinical heterogeneity.

Table 4 Proteogenomic analysis of subjects in the RLSC and CC groups performed to detect susceptibility genes/proteins

\begin{tabular}{|c|c|c|c|c|c|c|c|c|c|c|c|c|c|c|}
\hline \multirow{2}{*}{ Gene } & \multirow{2}{*}{ Chrom. } & \multirow{2}{*}{ Position } & \multirow{2}{*}{$\begin{array}{c}\text { Reference } \\
\text { allele }\end{array}$} & \multirow{2}{*}{$\begin{array}{l}\text { Sample } \\
\text { allele }\end{array}$} & \multirow{2}{*}{ Gene region } & \multirow{2}{*}{ Function } & \multirow{2}{*}{ cDNA variant } & \multirow{2}{*}{$\begin{array}{l}\text { Protein } \\
\text { variant }\end{array}$} & \multicolumn{3}{|c|}{ VAR reads $\%$} & \multicolumn{3}{|c|}{ ITRAQ } \\
\hline & & & & & & & & & RLSC-1 & RLSC-2 & CC-1 & $113: 121$ & $114: 121$ & 116:121 \\
\hline PRX & chr19 & 40903183 & $\mathrm{C}$ & $\mathrm{T}$ & exonic & missense SNV & G1076A & $\mathrm{R} 359 \mathrm{H}$ & 0.50 & . &. & 12.36 & 5.06 & 10.47 \\
\hline HNRNPDL & chr4 & 83350802 & $\mathrm{C}$ & A & exonic & missense SNV & G42T & L14F & 0.67 & . & . & 4.49 & 3.84 & 1.92 \\
\hline ITM2B & chr13 & 48807557 & A & $\mathrm{G}$ & exonic & missense SNV & A61G & $\mathrm{K} 21 \mathrm{E}$ & 0.47 & & . & 1.87 & 1.77 & 1.27 \\
\hline FYCO1 & chr3 & 45972579 & G & A & exonic & missense SNV & $\mathrm{C} 4235 \mathrm{~T}$ & P1412L & 0.60 & . & . & 0.85 & 0.96 & 1.02 \\
\hline BFSP2 & chr3 & 133119040 & G & A & exonic & missense SNV & G113A & $\mathrm{S} 38 \mathrm{~N}$ & 0.62 & . & . & 0.59 & 0.52 & 0.82 \\
\hline FASN & $\operatorname{chr} 17$ & 80048332 & $\mathrm{C}$ & $\mathrm{T}$ & exonic & missense SNV & G1789A & E597K & 0.47 & . & . & 0.32 & 0.34 & 1.07 \\
\hline TNPO1 & chr5 & 72173169 & $\mathrm{C}$ & $\mathrm{T}$ & $\begin{array}{l}\text { exonic; } \\
\text { splicing }\end{array}$ & missense SNV & C916T & P306S & 0.50 & . & . & 0.21 & 0.25 & 1.38 \\
\hline KRT10 & chr17 & 38975103 & A & $\mathrm{Aa}$ & exonic & $\begin{array}{l}\text { nonframeshift } \\
\text { insertion }\end{array}$ & 1683_1684insc & S562delinsd & 0.62 & 0.52 & 0.56 & 0.19 & 0.28 & 1.02 \\
\hline WFS1 & chr4 & 6302783 & A & $\mathrm{C}$ & exonic & missense SNV & A1261C & $\mathrm{I} 421 \mathrm{~L}$ & 0.43 & & . & 7.05 & 5.40 & 5.55 \\
\hline WFS1 & chr4 & 6290847 & $\mathrm{C}$ & $\mathrm{T}$ & exonic & missense SNV & $\mathrm{C} 449 \mathrm{~T}$ & $\mathrm{~A} 150 \mathrm{~V}$ & . & 0.44 & . & 7.05 & 5.40 & 5.55 \\
\hline GSN & chr 9 & 124089716 & $\mathrm{~Tb}$ & $\mathrm{~T}$ & exonic & frameshift deletion & 1872_1915del & $\mathrm{V} 624 \mathrm{fs}$ & . & 0.52 & . & 5.20 & 1.26 & 3.50 \\
\hline AGL & chr1 & 100327889 & $\mathrm{~T}$ & $\mathrm{~A}$ & exonic & missense SNV & $\mathrm{T} 319 \mathrm{~A}$ & L107M & . & 0.46 & . & 0.71 & 0.54 & 0.76 \\
\hline $\mathrm{CA} 2$ & chr8 & 86377590 & $\mathrm{C}$ & A & exonic & missense SNV & $\mathrm{C} 124 \mathrm{~A}$ & $\mathrm{P} 42 \mathrm{~T}$ & . & & 0.57 & 0.58 & 0.75 & 4.25 \\
\hline ALDH3A2 & chr17 & 19564572 & $\mathrm{C}$ & G & $\begin{array}{l}\text { exonic; } \\
\text { splicing }\end{array}$ & missense SNV & C931G & $\mathrm{R} 311 \mathrm{G}$ & . & 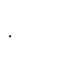 & 0.39 & 11.07 & 11.07 & 3.91 \\
\hline DPM1 & chr20 & 49552758 & $\mathrm{C}$ & $\mathrm{T}$ & exonic & missense SNV & G536A & $\mathrm{C} 179 \mathrm{Y}$ & . & & 0.53 & 12.13 & 7.18 & 2.01 \\
\hline UCHL1 & chr4 & 41259620 & $\mathrm{C}$ & $\mathrm{T}$ & splicing & . & & & . & & 0.27 & 0.08 & 0.14 & 0.45 \\
\hline CRYGD & chr2 & 208989018 & $\mathrm{G}$ & $\mathrm{T}$ & exonic & missense SNV & $\mathrm{C} 70 \mathrm{~A}$ & $\mathrm{P} 24 \mathrm{~T}$ & . & & 0.49 & 0.03 & 0.05 & 0.17 \\
\hline
\end{tabular}

Green represents the up-regulated proteins and red indicates the down-regulated proteins 
Protein Fold Change

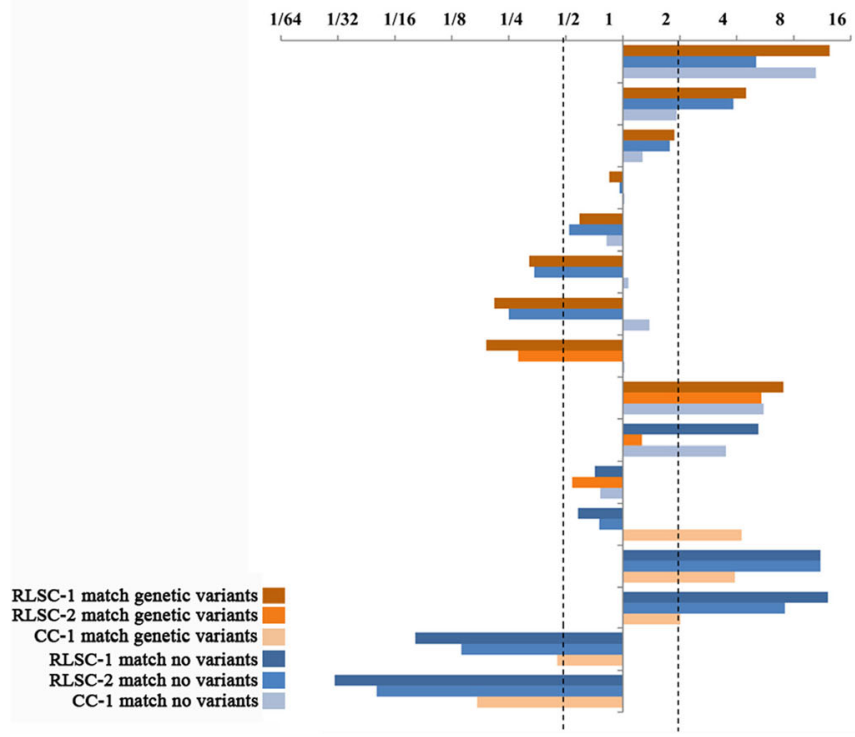

Genetic Variant reads\%

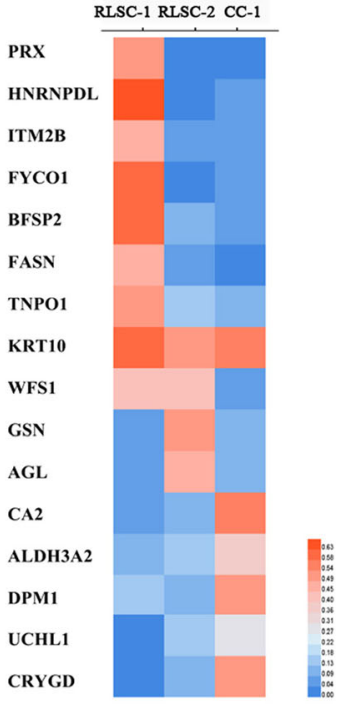

Fig. 6 Proteogenomic characterization of subjects in the RLSC and CC groups performed to detect susceptibility genes/proteins

\section{Conclusions}

These findings revealed that regenerative lens proteome composition was distinct from that of others. Cataractogenesis shares common final pathways with diverse upstream regulation of cataractogenesis in different physiopathological states. The proteogenomic analysis narrowed the list of candidate susceptibility genes/proteins, showing translational potential for detecting candidate causal molecules in precision medicine.

\section{Additional files}

Additional file 1: Table S1. Identification and quantification of total proteins across the RLSC, CC and ARC groups. (XLSX $217 \mathrm{~kb}$ )

Additional file 2: Table S2. Identification of genetic variants and filtering analysis for the RLSC and CC groups. (XLSX $22 \mathrm{~kb}$ )

\section{Abbreviations}

ARC: Age-related cataract; CC: Congenital cataract; ECCE: Extracapsular cataract extraction; GO: Gene Ontology; iTRAQ: Isobaric tagging for relative and absolute protein quantification; KEGG: Kyoto Encyclopedia of Genes and Genomes; LECs: Lens epithelial stem cells; RLSC: Regenerative lenses with secondary cataract; STRING: Search Tool for the Retrieval of Interacting Genes/Proteins; WES: Whole Exome Sequencing

\section{Funding}

This study was supported by the Ministry of Science and Technology of China Grants (973 program, 2015CB964600), the Key Research Plan for the National Natural Science Foundation of China in Cultivation Project (No. 91546101), the National Natural Science Foundation of China (No. 81300750), the Guangdong Province Universities and Colleges Youth Pearl River Scholar Funded Scheme (Haotian Lin), the Outstanding Young Teacher Cultivation Projects in Guangdong Province (No. YQ2015006), the Guangdong Provincial Natural Science Foundation for Distinguished Young Scholars of China (No. 2014A030306030), the Youth Science and Technology Innovation Talents Funds in a Special Support Plan for High Level Talents in Guangdong Province (No. 2014TQ01R573), the Young Teacher Top-Support project of Sun Yat-sen University (No. 2015ykzd11) and the
Fundamental Research Funds of the State Key Laboratory of Ophthalmology (2017QN04). The sponsor or funding organization had no role in the design or conduct of this research.

Availability of data and materials

All the raw data supporting the conclusions of this article is available within the article.

\section{Authors' contributions}

$H T L, Y Z L, X H W$ and ZZL designed the research. XHW, ZZL, XYZ, DNW, EPL, $J H W, W T L, W Y L, Q Z C, K H H$ and WRC conducted the experiments. XHW, ZZL, DNW, EPL, JHW and WTL collected the data. XHW, ZZL, XYZ, QZC, KHH, and WRC analyzed the data. XHW drafted the manuscript. HTL critically revised the manuscript. All authors discussed the results and commented on the manuscript. All authors read and approved the final manuscript.

Ethics approval and consent to participate

The study protocol conformed to the ethical guidelines of the 1975 Declaration of Helsinki and was approved by the Ethical Review Committee of ZOC. Written informed consent was obtained from all subjects (or from at least one guardian of each participating child).

\section{Consent for publication}

Not applicable.

\section{Competing interests}

The authors declare that they have no competing interests.

\section{Publisher's Note}

Springer Nature remains neutral with regard to jurisdictional claims in published maps and institutional affiliations.

\section{Author details}

${ }^{1}$ State Key Laboratory of Ophthalmology, Zhongshan Ophthalmic Center, Sun Yat-sen University, 54\# Xianlie Road, Guangzhou, Guangdong 510060, China. ${ }^{2}$ Department of Pharmacology, Zhongshan School of Medicine, Sun Yat-sen University, Guangzhou, Guangdong 510080, China. 
Received: 4 April 2017 Accepted: 4 December 2017

Published online: 19 December 2017

\section{References}

1. Lynnerup N, Kjeldsen H, Heegaard S, Jacobsen C, Heinemeier J. Radiocarbon dating of the human eye lens crystallines reveal proteins without carbon turnover throughout life. PLoS One. 2008;3(1):e1529.

2. Resnikoff S, Keys TU. Future trends in global blindness. Indian J Ophthalmol. 2012;60(5):387-95.

3. Hains PG, Truscott RJ. Proteome analysis of human foetal, aged and advanced nuclear cataract lenses. Proteomics Clin Appl. 2008;2(12):1611-9.

4. Takemoto L, Sorensen CM. Protein-protein interactions and lens transparency. Exp Eye Res. 2008;87(6):496-501.

5. Limburg H, Gilbert C, Hon do N, Dung NC, Hoang TH. Prevalence and causes of blindness in children in Vietnam. Ophthalmology. 2012;119(2):355-61.

6. Lin $\mathrm{H}$, Ouyang $\mathrm{H}$, Zhu J, Huang $\mathrm{S}$, Liu Z, Chen S, Cao G, Li G, Signer RA, Xu $Y$, et al. Lens regeneration using endogenous stem cells with gain of visual function. Nature. 2016;531(7594):323-8.

7. Wenke JL, McDonald WH, Schey KL. Spatially directed proteomics of the human lens outer cortex reveals an intermediate filament switch associated with the remodeling zone. Invest Ophthalmol Vis Sci. 2016;57(10):4108-14.

8. Truscott RJ, Comte-Walters S, Ablonczy Z, Schwacke JH, Berry Y, Korlimbinis A, Friedrich MG, Schey KL. Tight binding of proteins to membranes from older human cells. Age. 2011;33(4):543-54.

9. Kyselova Z. Mass spectrometry-based proteomics approaches applied in cataract research. Mass Spectrom Rev. 2011;30(6):1173-84.

10. Wu WW, Wang G, Baek SJ, Shen RF. Comparative study of three proteomic quantitative methods, DIGE, CICAT, and iTRAQ, using 2D gel- or LC-MALDI TOF/TOF. J Proteome Res. 2006;5(3):651-8.

11. Sanders SJ, Murtha MT, Gupta AR, Murdoch JD, Raubeson MJ, Willsey AJ Ercan-Sencicek AG, DiLullo NM, Parikshak NN, Stein JL, et al. De novo mutations revealed by whole-exome sequencing are strongly associated with autism. Nature. 2012;485(7397):237-41.

12. Lohr JG, Adalsteinsson VA, Cibulskis K, Choudhury AD, Rosenberg M Cruz-Gordillo P, Francis JM, Zhang CZ, Shalek AK, Satija R, et al. Wholeexome sequencing of circulating tumor cells provides a window into metastatic prostate cancer. Nat Biotechnol. 2014;32(5):479-84.

13. da Huang W, Sherman BT, Lempicki RA. Systematic and integrative analysis of large gene lists using DAVID bioinformatics resources. Nat Protoc. 2009;4(1):44-57.

14. Zhou HY, Yan H, Wang LL, Yan WJ, Shui YB, Beebe DC. Quantitative proteomics analysis by ITRAQ in human nuclear cataracts of different ages and normal lens nuclei. Proteomics Clin Appl. 2015;9(7-8):776-86

15. Wu $X$, Long E, Lin H, Liu Y. Prevalence and epidemiological characteristics of congenital cataract: a systematic review and meta-analysis. Sci Rep. 2016;6:28564.

16. Su S, Liu P, Zhang H, Li Z, Song Z, Zhang L, Chen S. Proteomic analysis of human age-related nuclear cataracts and normal lens nuclei. Invest Ophthalmol Vis Sci. 2011;52(7):4182-91.

17. Yousefi R, Javadi S, Amirghofran S, Oryan A, Moosavi-Movahedi AA. Assessment of structure, stability and aggregation of soluble lens proteins and alpha-crystallin upon non-enzymatic glycation: the pathomechanisms underlying cataract development in diabetic patients. Int J Biol Macromol. 2016;82:328-38.

18. Moreau KL, King JA. Cataract-causing defect of a mutant gamma-crystallin proceeds through an aggregation pathway which bypasses recognition by the alpha-crystallin chaperone. PLoS One. 2012;7(5):e37256.

19. Iwasaki N, David LL, Shearer TR. Crystallin degradation and insolubilization in regions of young rat lens with calcium ionophore cataract. Invest Ophthalmol Vis Sci. 1995:36(2):502-9.

20. Pichi F, Lembo A, Serafino M, Nucci P. Genetics of congenital cataract. Dev Ophthalmol. 2016;57:1-14.

21. Li L, Zhang H, Wang W, Hong Y, Wang J, Zhang S, Xu S, Shu Q, Li J, Yang F, et al. Comparative proteomics reveals abnormal binding of ATGL and dysferlin on lipid droplets from pressure overload-induced dysfunctional rat hearts. Sci Rep. 2016;6:19782.

22. Mertins P, Mani DR, Ruggles KV, Gillette MA, Clauser KR, Wang P, Wang X Qiao JW, Cao S, Petralia F, et al. Proteogenomics connects somatic mutations to signalling in breast cancer. Nature. 2016;534(7605):55-62.

\section{Submit your next manuscript to BioMed Central and we will help you at every step:}

- We accept pre-submission inquiries

- Our selector tool helps you to find the most relevant journal

- We provide round the clock customer support

- Convenient online submission

- Thorough peer review

- Inclusion in PubMed and all major indexing services

- Maximum visibility for your research

Submit your manuscript at www.biomedcentral.com/submit 